\title{
LARGE-SCALE FLOWS IN THE LOCAL UNIVERSE
}

\author{
D.S. MATHEWSON and V.L. FORD \\ Mount Stromlo and Siding Spring Observatories, \\ The Australian National University, ACT 2611, Australia
}

\begin{abstract}
Peculiar velocity measurements of 2500 southern spiral galaxies show large-scale flows in the direction of the Hydra-Centaurus clusters which fully participate in the flow themselves. The flow is not uniform over this region and seems to be associated with the denser regions which participate in the flow of amplitude about $400 \mathrm{~km} / \mathrm{s}$. In the less dense regions the flow is small or non-existent. This makes the flow quite asymmetric and inconsistent with that expected from large-scale, parallel streaming flow that includes all galaxies out to $6000 \mathrm{~km} / \mathrm{s}$ as previously thought. The flow cannot be modelled by a Great Attractor at $4300 \mathrm{~km} / \mathrm{s}$ or the Centaurus clusters at $3500 \mathrm{~km} / \mathrm{s}$. Indeed, from the density maps derived from the redshift surveys of "optical" and IRAS galaxies, it is difficult to see how the mass concentrations can be responsible particularly as they themselves participate in the flow. These results bring into question the generally accepted reason for the peculiar velocities of galaxies that they arise solely as a consequence of infall into the dense regions of the universe. To the N. of the Great Attractor region, the flow increases and shows no sign of diminishing out to the redshift limit of $8000 \mathrm{~km} / \mathrm{s}$ in this direction. We may have detected flow in the nearest section of the Great Wall.
\end{abstract}

\section{Introduction}

The results of a typical N-body simulation of the formation of structure in our Universe and the predicted peculiar velocity field using the standard CDM theory are shown in Figure 2 of Nusser et al. (1991). As expected the flows are towards the mass-centers produced by their simulation. Their working hypotheses concern the nature of the primordial density fluctuations, the process of gravitational instability, the value of $\Omega / b$ where $b$ is the biassing factor and that the galaxy and mass-density fluctuations are proportional at each point.

These hypotheses can be tested by measurements of, 1) the cosmic microwave background (CMB), its irregularities and dipole, 2) the distribution of galaxies in redshift-space from which the galaxy-density field may be obtained to make peculiar velocity (Vpec) predictions and 3) the Vpec of galaxies and the construction using POTENT (Bertschinger \& Dekel 1989) of the mass-density field. A comparison of the density fields obtained from redshifts to those obtained from Vpec is the crucial test of the current theories of the evolution of the universe.

The 1990s have seen the disappearance of the concept of the Great Attractor (GA) introduced by Lynden-Bell et al. (1988) as the mystery object responsible for the observed flows in the local universe and the reintroduction of large-scale bulk flows of amplitude about $400 \mathrm{~km} / \mathrm{s}$. What drives these bulk flows is the subject of much debate. 
Willick (1990) detected a flow of galaxies from the direction of Perseus-Pisces toward the Local Group. Mathewson, Ford and Buchhorn (1992a, b) from the Vpec measurements of 1355 southern spiral galaxies, found that there was evidence of a bulk flow of about $600 \mathrm{~km} / \mathrm{s}$ towards the direction of the $\mathrm{GA}$ region on a scale of at least $6000 \mathrm{~km} / \mathrm{s}$. This, when added to the bulk flow of $450 \mathrm{~km} / \mathrm{s}$ found by Willick in the opposite part of the sky, suggested that there is bulk flow in the supergalactic plane over very large scales greater than $13000 \mathrm{~km} / \mathrm{s}$. More recently Courteau et al. (1993), using a new set of Tully-Fisher (TF) distances for northern spirals and the data from Mathewson et al. (1992b), support the previous findings and conclude that there is a large-scale, parallel streaming flow of $360 \mathrm{~km} / \mathrm{s}$ towards $1=294^{\circ}, \mathrm{b}=0^{\circ}$ that includes all galaxies out to at least $6000 \mathrm{~km} / \mathrm{s}$ radius around the Local Group.

In an attempt to probe deeper into the local universe to see if the scale of this bulk flow can be determined, we extended the survey of 1355 galaxies by Mathewson et al. (1992b) and made Vpec measurements of an additional 1200 galaxies. The combined data set of about 2500 galaxies has been used to examine these large-scale flows and the preliminary results are presented here (also see Mathewson \& Ford 1994).

\section{The Data}

The extended sample of spiral galaxies was selected from the ESO-Uppsala Catalog of types $\mathrm{Sb}-\mathrm{Sc}$, diameters between 1'.0 and 1'.6, velocities in general between $4000 \mathrm{~km} / \mathrm{s}$ and $14000 \mathrm{~km} / \mathrm{s}$ inclinations greater than $40^{\circ}$ and $\mathrm{lbl}>11^{\circ}$. Altogether over 1200 galaxies were observed. These included 90 galaxies with redshifts less than $8000 \mathrm{~km} / \mathrm{s} \mathrm{N}$. of the $\mathrm{GA}$ region $\left(1=250^{\circ}\right.$ to $360^{\circ}, \mathrm{b}=45^{\circ}$ to $\left.70^{\circ}\right)$ from a list kindly supplied by Dr John Huchra.

The $\mathrm{H} \alpha$ rotation curves of the spiral galaxies were measured with the $2.3 \mathrm{~m}$ telescope at Siding Spring Observatory using the same procedures described in Mathewson et al (1992b). Photometry was obtained in the Kron-Cousins I passband with a GEC CCD on the $1 \mathrm{~m}$ telescope. The I magnitudes were found using methods outlined in the first survey. The "zero-velocity" HI was observed in the direction of each galaxy with the Parkes radiotelescope and used to measure the extinction in our Galaxy following Burstein and Heiles (1978).

The complete data set when combined with the 1355 galaxies measured by Mathewson et al (1992b) consists of about 2500 galaxies with 1200 galaxies lying in the direction of the GA region defined as $260^{\circ}<1<360^{\circ},-40^{\circ}<b<45^{\circ}$. The aim is to investigate the flow already detected in the direction of the GA with this combined data set.

\section{The TF Relation and Selection Bias}

The method is to assume that the galaxies outside the GA region (apart from galaxies to the $\mathrm{N}$. of $\mathrm{b}=45^{\circ}$ and in the Perseus-Pisces region) are at sufficiently large angles to the flow that their velocities only reflect pure Hubble flow i.e., their redshifts are their distances. These galaxies in this control zone are then used to calibrate the TF relation which is used to estimate the Vpec of the galaxies in the direction of the flow.

The luminosity distribution of the sample changes with increasing redshift, showing that observational selection bias exists. The peak magnitude progressively moves to brighter values as the redshift increases. This, coupled with the dispersion $(\sigma=$ 0.44 ) of the TF relation combines to make the absolute magnitude of galaxies a function of redshift as well as rotational velocity, Vrot. (also see Federspiel et al. 1994). The dispersion of the TF relation increases markedly for Vrot $<63 \mathrm{~km} / \mathrm{s}$ and these galaxies have been omitted from the analysis. 


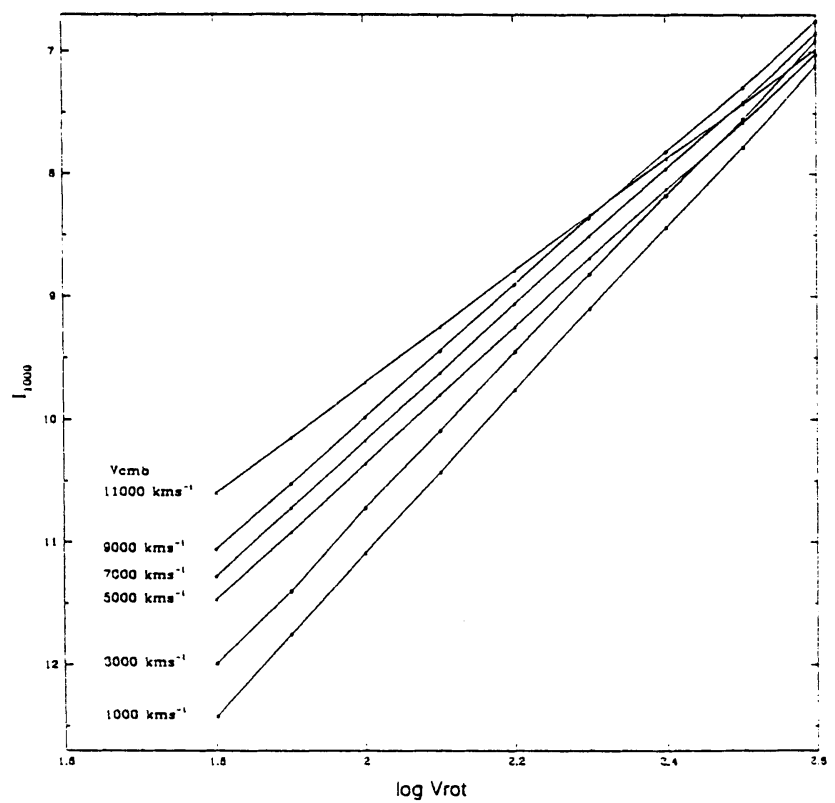

Fig. 1. The Tully-Fisher relation as a function of redshift $(\mathrm{Vcmb})$ for the galaxies in the control region. I 1000, the magnitude of the galaxies at a distance of $1000 \mathrm{~km} / \mathrm{s}$, is plotted against log Vrot, the velocity of rotation for galaxies in redshift bins $2000 \mathrm{~km} / \mathrm{s}$ wide centred on the values given at the bottom of each plot.

Using the galaxies in the control zone, Figure 1 was constructed, which gives I 1000 as a function of Vrot and redshift (distance). I 1000 is the magnitude of the galaxies at a distance of $1000 \mathrm{~km} / \mathrm{s}$. In this paper all redshifts are relative to the cosmic microwave background, Vcmb. The values of I 1000 for the galaxies in the GA region are found using Figure 1. As the luminosity distribution of galaxies in the GA region is very similar to those in the control zone, this process should remove most of the selection bias and allow measurement of the Vpec of galaxies in the GA region relative to the control zone. The accuracy of an individual measurement of distance is about $22 \%$.

To improve the accuracy of the detection of large-scale flows, the galaxies were divided into redshift bins of $1000 \mathrm{~km} / \mathrm{s}$ and averaged. With the present sample, this procedure would allow flows of about $300 \mathrm{~km} / \mathrm{s}$ or more to be detected at distances of $11000 \mathrm{~km} / \mathrm{s}$. At greater distances, the fluctuations increase rapidly due to errors which increase with distance and small number statistics and the results have small significance.

As a check, Figure 2 plots Vpec vs Vcmb for the 1100 galaxies in the control zone. The galaxies were divided into redshift bins of $1000 \mathrm{~km} / \mathrm{s}$ and averaged. The averaged points lie close to the $\mathrm{Vpec}=0$ line with mean value $-57 \pm 49 \mathrm{~km} / \mathrm{s}$ consistent with no net flow. 


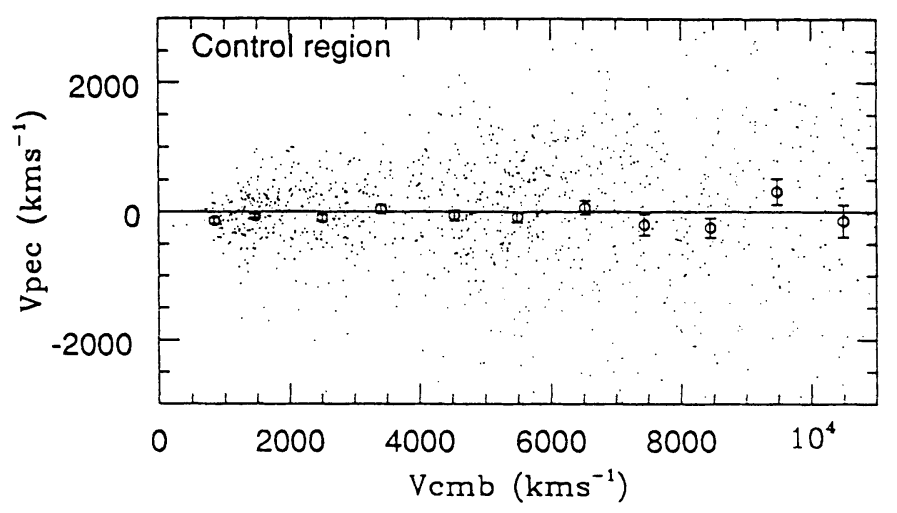

Fig. 2. Vpec vs Vcmb for the control zone which is the region outside the Great Attractor box in Fig. 3 (apart from galaxies to the N. of that box and in the Perseus-Pisces region). This is assumed to be a region of quiet Hubble flow. The open circles are the averages of the galaxies (dots) in intervals of $1000 \mathrm{~km} / \mathrm{s}$ Vcmb. A total of 1100 galaxies are plotted.

\section{Results}

Figure 3 shows the distribution in galactic longitude and latitude of the galaxies used in this analysis. The GA region has been divided into four areas and Figure 4 shows Vpec vs Vcmb plots for the galaxies within them. The galaxies have been placed in $1000 \mathrm{~km} / \mathrm{s}$ bins of Vcmb and averages taken. The three areas in Centaurus (Fig. 3, area a), Hydra (Fig. 3, area b) and Pavo (Fig. 3, area c) show well defined flows of approximately 300 $400 \mathrm{~km} / \mathrm{s}$ out to distances of $6000-7000 \mathrm{~km} / \mathrm{s}$ when the flow vanishes into the increasing noise at greater distances. The amplitudes of the flows in each area are marked against each box in Figure 3. Figure 5 shows the distribution in redshift space of the galaxies in the GA region. The flow appears to be associated with the denser regions (Figs. 3 and 5 and Fig. 1 of Hudson 1994) which participate in the flow. In the less dense areas in the GA region, i.e., beyond $6500 \mathrm{~km} / \mathrm{s}$ and in the direction of area $\mathrm{d}$ in Fig. 3, the flow is not so well defined.

Figure 6 shows the combined plot of Vpec vs Vcmb for the three areas in the GA region showing flow. The galaxies have been divided into bins of $1000 \mathrm{~km} / \mathrm{s} \mathrm{Vcmb}$ and averages taken in each bin denoted by the open circles. The flow of $370 \pm 16 \mathrm{~km} / \mathrm{s}$ is clearly defined up to $6000 \mathrm{~km} / \mathrm{s}$ but from $\mathrm{Vcmb}=7000 \mathrm{~km} / \mathrm{s}$ to $11000 \mathrm{~km} / \mathrm{s}$, the mean value of $\mathrm{Vpec}$ is only $3 \pm 128 \mathrm{~km} / \mathrm{s}$ which is consistent with no flow. As the control region (Fig. 2) has Vpec effectively zero from $\mathrm{Vcmb}=0$ to $11000 \mathrm{~km} / \mathrm{s}$ and if it can be regarded as a region of quiet Hubble flow, then the above estimated flow values represent the real flow values of the GA region relative to the CMB. Our flow value of $370 \mathrm{~km} / \mathrm{s}$ agrees closely with that found by Courteau et al (1993) over the same distance range who used as a control, a region almost diametrically opposed to ours.

Figure 7 shows the plot of Vpec vs Vcmb for the region $1=250^{\circ}-360^{\circ}, b=45^{\circ}$ $70^{\circ}$, to the N. of the GA region (see Fig. 3). There is strong bulk flow of $489 \pm 61 \mathrm{~km} / \mathrm{s}$ which shows no sign of diminishing out to $8000 \mathrm{~km} / \mathrm{s}$, the redshift limit of the sample in this direction. 


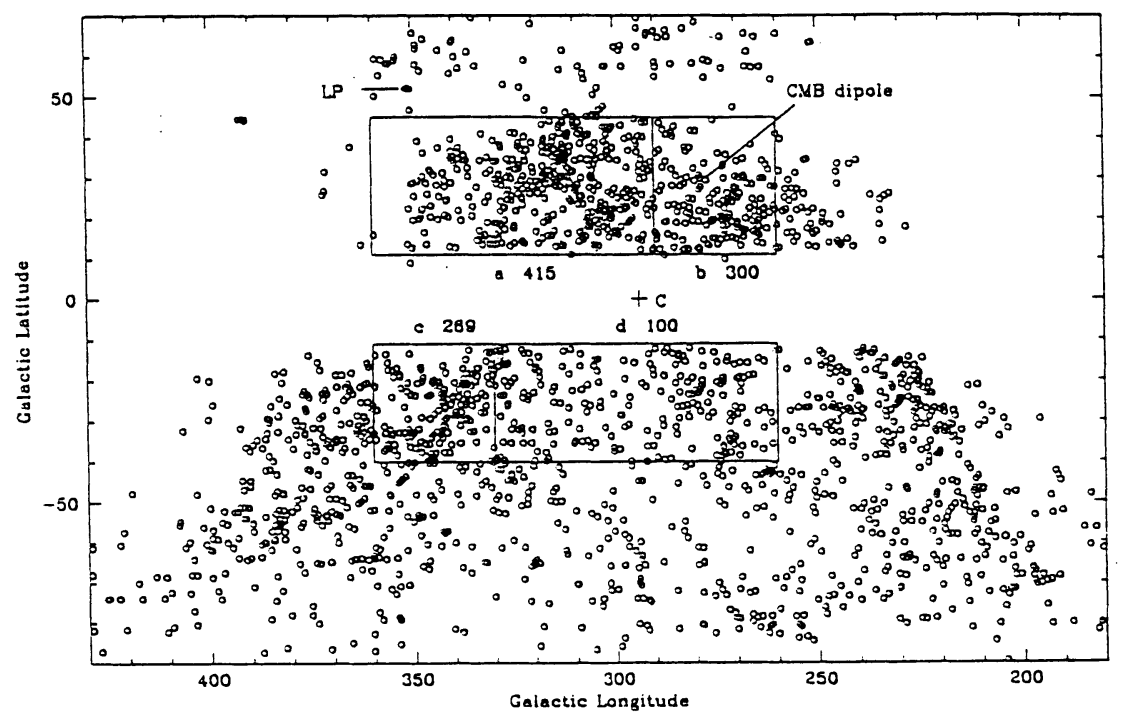

Fig. 3. The distribution in galactic longitude and latitude of the 2500 southern spiral galaxies used in the analysis of peculiar velocities. The GA region is outlined and split into four areas against which is indicated the amplitude of the flow derived using Fig. 4. The directions of the CMB dipole and the bulk flow directions denoted C and LP of Courteau et al (1993) and Lauer and Postman (1994) respectively, are shown.
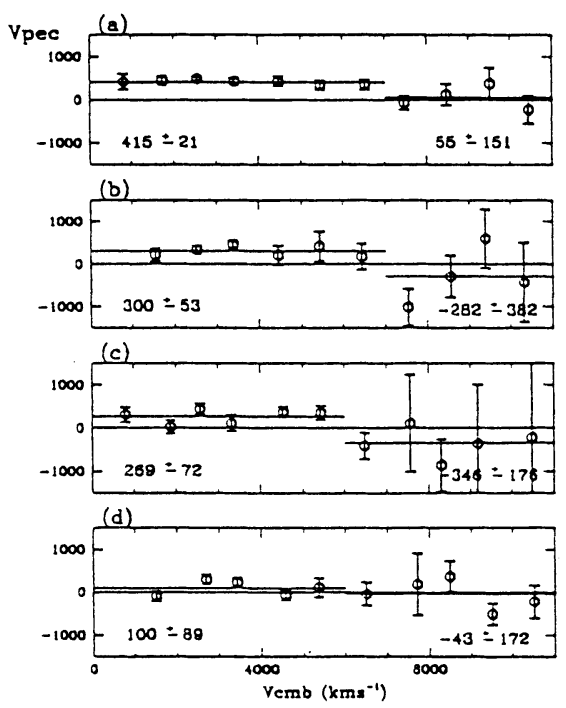

Fig. 4. Vpec vs Vcmb for the four areas in the GA region shown in Fig. 3. Each plot is labelled with a letter to identify which area it refers to in Fig. 3. The open circles give the average Vpec of the galaxies in intervals of $1000 \mathrm{~km} / \mathrm{s} \mathrm{Vcmb}$. The mean of Vpec above and below $\mathrm{Vcmb}=7000 \mathrm{~km} / \mathrm{s}$ for (a) and (b) and $6000 \mathrm{~km} / \mathrm{s}$ for (c) and (d) are shown on the RHS and LHS of each plot respectively. The amplitude of the flows is shown against each box in Fig. 3 . 


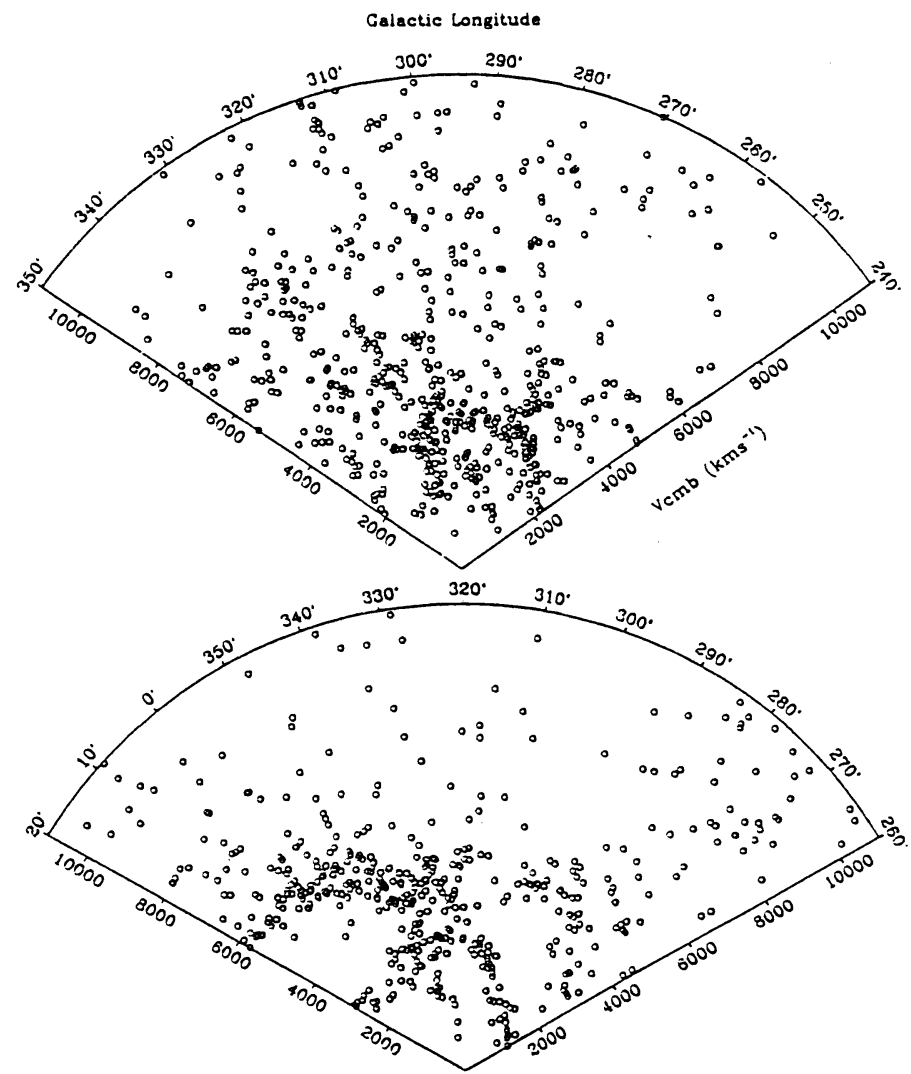

Fig. 5. The distribution in redshift-space for the galaxies in the GA region; a) +ve latitudes, b) -ve latitudes.

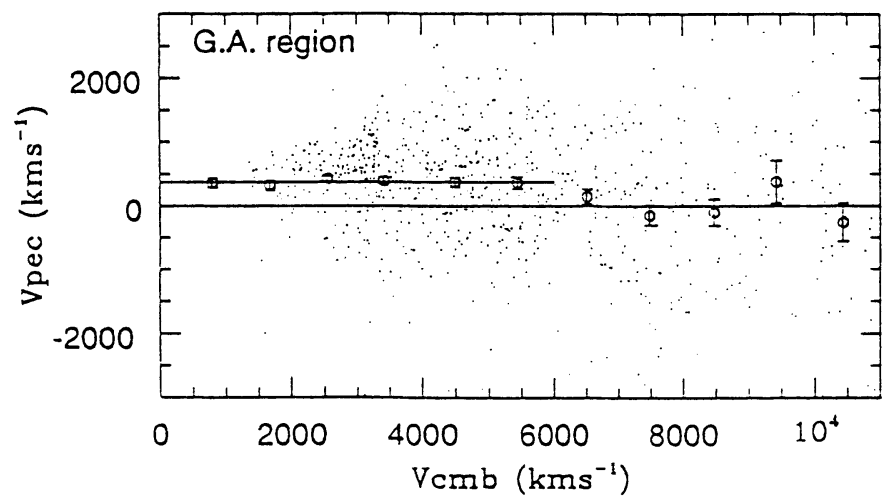

Fig. 6. The combined Vpec vs Vcmb plot for the three areas (a, b, c) which show strong flow in the GA region. The open circles give the average $V$ pec of galaxies (dots) in intervals of $1000 \mathrm{~km} / \mathrm{s} \mathrm{Vcmb}$. The mean of $\mathrm{Vpec}$ below and above $\mathrm{Vcmb}=6000 \mathrm{~km} / \mathrm{s}$ is $370 \pm 16 \mathrm{~km} / \mathrm{s}$ and $3 \pm 128 \mathrm{~km} / \mathrm{s}$ respectively. 


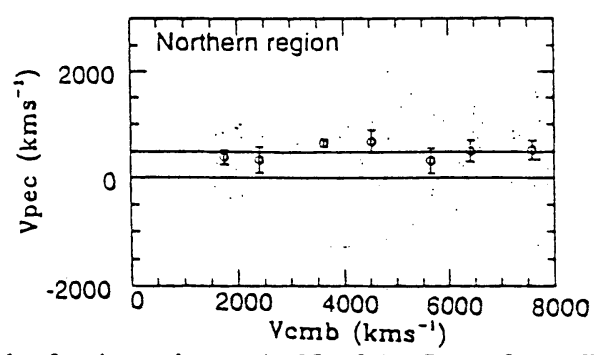

Fig. 7. Vpec vs Vcmb plot for the region to the $\mathrm{N}$. of the $\mathrm{GA}$ region. The open circles give the average $\mathrm{Vpec}$ of the galaxies (dots) in intervals of $1000 \mathrm{~km} / \mathrm{s} \mathrm{Vcmb}$. The redshift limit of the sample in this direction is $8000 \mathrm{~km} / \mathrm{s}$. The mean Vpec over this redshift range is $489 \pm 61 \mathrm{~km} / \mathrm{s}$.

\section{Discussion}

Because the flow in the GA region is associated with its denser regions, it is natural to suspect that the TF relation is dependent on environment which may create the illusion of a flow. However the flow would seem to be genuine because:-

1. northern hemisphere observers, using the TF relation, have found that the PerseusPisces supercluster is flowing towards us, i.e., in the opposite direction to the GA region which is moving away. If there was some effect artificially producing flows, it would need to change sign with direction, which is most unlikely,

2. Dressler et al (1987), using the $\sigma$-D relation for ellipticals, have shown outflow in the Centaurus-Hydra region of about the same amplitude as we have shown using the TF relation for spirals. However the $\sigma-D$ and TF relations are quite different physical relations, the former tells us about galaxy equilibrium while the latter tells us about galaxy formation. M/L constancy does enter both relations but the stellar populations in the two galaxy types are quite different so it would be remarkable if $\mathrm{M} / \mathrm{L}$ variations in both types were the same,

3. the strongest flow in the GA region of $450 \mathrm{~km} / \mathrm{s}$ is centred on $1=310^{\circ}, b=30^{\circ}$ in Centaurus only $30^{\circ}$ from the CMB dipole at $l=276^{\circ}, b=30^{\circ}$ (Kogut et al 1993) which is itself in a region of strong flow (Fig 3). This provides independent support for our Vpec measurements as there is no doubt now that the dipole is due to a Vpec of the Local Group of about $600 \mathrm{~km} / \mathrm{s}$ (Fixsen et al 1994).

It is very difficult to model the observed asymmetric flow with a bulk flow of amplitude $360 \mathrm{~km} / \mathrm{s}$ toward $\mathrm{l}=294^{\circ}, \mathrm{b}=0^{\circ}$ over a distance of $6000 \mathrm{~km} / \mathrm{s}$ (Courteau et al 1993) when the adjacent area d, Fig. 3, shows a flow of only $100 \pm 89 \mathrm{~km} / \mathrm{s}$ instead of a predicted flow of at least $300 \mathrm{~km} / \mathrm{s}$. Also there is no sign of the expected "S-curve" from a GA at $4200 \mathrm{~km} / \mathrm{s}$ in the direction $1=307^{\circ}, b=9^{\circ}$ (Lynden-Bell et al 1988). Likewise there appears little chance of salvaging the GA by calling it Hydra-Centaurus (Federspiel et al 1994 ) because these clusters at $3500 \mathrm{~km} / \mathrm{s}$ do not have an overly large effect on the flow in which they themselves fully participate. Courteau et al (1993) suggested that largescale, low amplitude density fluctuations are responsible for the flow of about $400 \mathrm{~km} / \mathrm{s}$ from the Perseus-Pisces region to the GA region, over a distance of $12000 \mathrm{~km} / \mathrm{s}$. However this does not explain the fall to zero around $6500 \mathrm{~km} / \mathrm{s}$ in the GA region (Fig. 4) nor the asymmetric nature of the flow about its apex, nor the large amplitude of the flow compared with the apparent small effect on the flow of the mass concentrations, e.g., Centaurus. Shapley 8 at $14000 \mathrm{~km} / \mathrm{s}$ does not seem to exert much power on the 
flow as at $11000 \mathrm{~km} / \mathrm{s}$, the flow is still small. It is apparently not the Giant Attractor as proposed by Scaramella et al (1989).

Hudson (1994) derived the density field out to a depth of $8000 \mathrm{~km} / \mathrm{s}$ using redshift surveys of galaxies selected from ESO and UGC catalogs and predicted the peculiar velocities of the galaxies. He finds that the Centaurus-Hydra-Virgo and Pavo supercluster complex is not primarily responsible for the large streaming motions of galaxies. Hudson believes that most of the bulk motion of the $405 \mathrm{~km} / \mathrm{s}$ which is required so as to agree with the predicted motion of the Local Group, is due to sources beyond $8000 \mathrm{~km} / \mathrm{s}$. However the next large mass concentration encountered in the flow direction is Shapley 8 at $14000 \mathrm{~km} / \mathrm{s}$ and this does not appear to have much effect on the flow.

It is not just a question of whether light traces mass but whether the standard theory of the formation of large-scale structure with the consequent Vpec flows is correct (c.f. Silk 1987). In this regard the main difficulty that the standard theory faces is to explain why the large visible mass centers of the Local Universe do not appear to produce large scale flows but instead fully participate in the flows themselves. This finding does not negate the result of Lauer and Postman (1994) of bulk flows of $689 \mathrm{~km} / \mathrm{s}$ out to distances of $15000 \mathrm{~km} / \mathrm{s}$ as flows may be a property of the denser regions in which are embedded their observed clusters. In this respect, it is intriguing that the bulk flow is strongest in the region $\mathrm{N}$. of the GA region and shows no sign of abating out to the survey limit in this direction of $8000 \mathrm{~km} / \mathrm{s}$ (Fig. 7). This region encompasses Lauer and Postman's flow direction of $1=343^{\circ}, b=52^{\circ}$, marked in Fig. 3 . It is interesting that the nearer parts of the Great Wall lie around $1=330^{\circ}, \mathrm{b}=70^{\circ}$ at a distance of about $7000 \mathrm{~km} / \mathrm{s}$ (Geller and Huchra, 1989 and also see Fig. 10 of Hudson, 1993). Has the flow of the Great Wall been detected? There is an obvious need for more observations in this area.

The authors thank the staff of Siding Spring Observatory for maintaining the telescopes in excellent condition throughout the observations.

\section{References}

Bertschinger, E. and Dekel, A.: 1989, ApJ. 336, L5.

Bursteain, D. and Heiles, C.: 1978, ApJ. 225, 40.

Courteau, S., Faber, S.M., Dressler, A. and Willick, J.A.: 1993, ApJ. 412, L51.

Dressler, A., Faber, S.M., Burstein, D., Davies, R.L., Lynden-Bell, D., Terlevich, R.J. and Wegner, F.: 1987, ApJ. 313, L37.

Federspiel, M., Sandage, A. and Tammann, G.A.: 1994, ApJ. 430, 29.

Fixsen, D.J. et al: 1994, ApJ. 420, 439.

Geller, M.J. and Huchra, J.P.: 1989, Science. 246, 857.

Hudson, M.J.: 1993, MNRAS. 265, 43.

Hudson, M.J.: 1994, MNRAS. 266, 475.

Kogut, A. et al: 1993, ApJ. 419, 1.

Lauer, T.R. and Postman, M.: 1994, ApJ. 425, 418.

Lynden-Bell, D., Faber, S.M., Burstein, D., Davies, R.L., Dressler, A., Terlevich, R.J. and Wegner, G.: 1988, ApJ. 326, 19.

Mathewson, D.S., Ford. V.L. and Buchhorn, M.: 1992a, ApJ. 389, L5.

Mathewson, D.S., Ford. V.L. and Buchhorn, M.: 1992b, ApJS. 81, 413.

Mathewson, D.S. and Ford, V.L.: 1994, ApJ (in press)

Nusser, A., Dekel, A., Bertschinger, E. and Blumenthal, G.R.: 1991, ApJ. 379, 6.

Scaramella, R., Baiesi-Pillastrini, G., Chincarini, G., Vettolani, G. and Zamorani, G.: 1989, Nature. 338, 562.

Silk, J.: 1987, "Observational Cosmology", IAU Symposium No. 124, p.391.

Willick, J.A.: 1990, ApJ. 351, L5. 